DESY $98-143$

ISSN $0418-9833$

September 1998

\title{
Forward Jet and Particle Production at HERA
}

\author{
H1 Collaboration
}

\begin{abstract}
Single particles and jets in deeply inelastic scattering at low $x$ are measured with the $\mathrm{H} 1$ detector in the region away from the current jet and towards the proton remnant, known as the forward region. Hadronic final state measurements in this region are expected to be particularly sensitive to QCD evolution effects. Jet crosssections are presented as a function of Bjorken- $x$ for forward jets produced with a polar angle to the proton direction, $\theta_{\text {jet }}$, in the range $7^{\circ}<\theta_{\text {jet }}<20^{\circ}$. Azimuthal correlations are studied between the forward jet and the scattered lepton. Charged and neutral single particle production in the forward region are measured as a function of Bjorken- $x$, in the range $5^{\circ}<\theta<25^{\circ}$, for particle transverse momenta larger than $1 \mathrm{GeV}$. QCD based Monte Carlo predictions and analytical calculations based on BFKL, CCFM and DGLAP evolution are compared to the data. Predictions based on the DGLAP approach fail to describe the data, except for those which allow for a resolved photon contribution.
\end{abstract}

Submitted to Nuclear Physics B 


\section{H1 Collaboration}

C. Adloff ${ }^{34}$, M. Anderson ${ }^{22}$, V. Andreev ${ }^{25}$, B. Andrieu ${ }^{28}$, V. Arkadov ${ }^{35}$, C. Arndt ${ }^{11}$, I. Ayyaz ${ }^{29}$, A. Babaev ${ }^{24}$, J. Bähr ${ }^{35}$, J. Bán ${ }^{17}$, P. Baranov ${ }^{25}$, E. Barrelett ${ }^{29}$, W. Bartel ${ }^{11}$, U. Bassler ${ }^{29}$, P. Bate ${ }^{22}$, M. Beck ${ }^{13}$, A. Beglarian ${ }^{11,40}$, O. Behnke ${ }^{11}$, H.-J. Behrend ${ }^{11}$, C. Beier ${ }^{15}$, A. Belousov ${ }^{25}$, Ch. Berger ${ }^{1}$, G. Bernardi ${ }^{29}$, G. Bertrand-Coremans ${ }^{4}$, P. Biddulph ${ }^{22}$, J.C. Bizot ${ }^{27}$, V. Boudry ${ }^{28}$, W. Braunschweig ${ }^{1}$, V. Brisson ${ }^{27}$, D.P. Brown ${ }^{22}$, W. Brückner ${ }^{13}$, P. Bruel ${ }^{28}$, D. Bruncko ${ }^{17}$, J. Bürger ${ }^{11}$, F.W. Büsser ${ }^{12}$, A. Buniatian ${ }^{32}$, S. Burke ${ }^{18}$, G. Buschhorn ${ }^{26}$, D. Calvet ${ }^{23}$, A.J. Campbell ${ }^{11}$, T. Carli ${ }^{26}$, E. Chabert ${ }^{23}$, M. Charlet ${ }^{4}$, D. Clarke $^{5}$, B. Clerbaux ${ }^{4}$, S. Cocks ${ }^{19}$, J.G. Contreras ${ }^{8,42}$, C. Cormack ${ }^{19}$, J.A. Coughlan ${ }^{5}$, M.-C. Cousinou ${ }^{23}$, B.E. $\mathrm{Cox}^{22}$, G. Cozzika ${ }^{10}$, J. Cvach ${ }^{30}$, J.B. Dainton ${ }^{19}$, W.D. Dau ${ }^{16}$, K. Daum ${ }^{39}$, M. David ${ }^{10}$, M. Davidsson ${ }^{21}$, A. De Roeck ${ }^{11}$, E.A. De Wolf ${ }^{4}$, B. Delcourt ${ }^{27}$, R. Demirchyan ${ }^{11,40}$, C. Diaconu ${ }^{23}$, M. Dirkmann ${ }^{8}$, P. Dixon ${ }^{20}$, W. Dlugosz ${ }^{7}$, K.T. Donovan ${ }^{20}$, J.D. Dowell ${ }^{3}$, A. Droutskoi ${ }^{24}$, J. Ebert ${ }^{34}$, G. Eckerlin ${ }^{11}$, D. Eckstein ${ }^{35}$, V. Efremenko ${ }^{24}$, S. Egli ${ }^{37}$, R. Eichler ${ }^{36}$, F. Eisele ${ }^{14}$, E. Eisenhandler ${ }^{20}$, E. Elsen ${ }^{11}$, M. Enzenberger ${ }^{26}$, M. Erdmann ${ }^{14}$, A.B. Fahr ${ }^{12}$, L. Favart ${ }^{4}$, A. Fedotov ${ }^{24}$, R. Felst ${ }^{11}$, J. Feltesse ${ }^{10}$, J. Ferencei ${ }^{17}$, F. Ferrarotto ${ }^{32}$, M. Fleischer ${ }^{8}$, G. Flügge ${ }^{2}$, A. Fomenko ${ }^{25}$, J. Formánek ${ }^{31}$, J.M. Foster ${ }^{22}$, G. Franke ${ }^{11}$, E. Gabathuler ${ }^{19}$, K. Gabathuler ${ }^{33}$, F. Gaede ${ }^{26}$, J. Garvey ${ }^{3}$, J. Gayler ${ }^{11}$, R. Gerhards ${ }^{11}$, S. Ghazaryan ${ }^{11,40}$, A. Glazov ${ }^{35}$, L. Goerlich ${ }^{6}$, N. Gogitidze ${ }^{25}$, M. Goldberg ${ }^{29}$, I. Gorelov ${ }^{24}$, C. Grab ${ }^{36}$, H. Grässler ${ }^{2}$, T. Greenshaw ${ }^{19}$, R.K. Griffiths ${ }^{20}$, G. Grindhammer ${ }^{26}$, T. Hadig ${ }^{1}$, D. Haidt ${ }^{11}$, L. Hajduk ${ }^{6}$, T. Haller ${ }^{13}$, M. Hampel ${ }^{1}$, V. Haustein ${ }^{34}$, W.J. Haynes ${ }^{5}$, B. Heinemann ${ }^{11}$, G. Heinzelmann ${ }^{12}$, R.C.W. Henderson ${ }^{18}$, S. Hengstmann ${ }^{37}$, H. Henschel ${ }^{35}$, R. Heremans ${ }^{4}$, I. Herynek ${ }^{30}$, K. Hewitt ${ }^{3}$, K.H. Hiller ${ }^{35}$, C.D. Hilton ${ }^{22}$, J. Hladký ${ }^{30}$, D. Hoffmann ${ }^{11}$, T. Holtom ${ }^{19}$, R. Horisberger ${ }^{33}$, V.L. Hudgson ${ }^{3}$, S. Hurling ${ }^{11}$, M. Ibbotson ${ }^{22}$, Ç. İssever ${ }^{8}$, H. Itterbeck ${ }^{1}$, M. Jacquet ${ }^{27}$, M. Jaffre ${ }^{27}$, D.M. Jansen ${ }^{13}$, L. Jönsson ${ }^{21}$, D.P. Johnson ${ }^{4}$, H. Jung ${ }^{21}$, H.K. Kästli ${ }^{36}$, M. Kander ${ }^{11}$, D. Kant ${ }^{20}$, M. Kapichine ${ }^{9}$, M. Karlsson ${ }^{21}$, O. Karschnik ${ }^{12}$, J. Katzy ${ }^{11}$, O. Kaufmann ${ }^{14}$, M. Kausch ${ }^{11}$, I.R. Kenyon ${ }^{3}$, S. Kermiche ${ }^{23}$, C. Keuker ${ }^{1}$, C. Kiesling ${ }^{26}$, M. Klein ${ }^{35}$, C. Kleinwort ${ }^{11}$, G. Knies ${ }^{11}$, J.H. Köhne ${ }^{26}$, H. Kolanoski ${ }^{38}$, S.D. Kolya ${ }^{22}$, V. Korbel ${ }^{11}$, P. Kostka ${ }^{35}$, S.K. Kotelnikov ${ }^{25}$, T. Krämerkämper ${ }^{8}$, M.W. Krasny ${ }^{29}$, H. Krehbiel ${ }^{11}$, D. Krücker ${ }^{26}$, K. Krüger ${ }^{11}$, A. Küpper ${ }^{34}$, H. Küster ${ }^{2}$, M. Kuhlen ${ }^{26}$, T. Kurča ${ }^{35}$, B. Laforge $^{10}$, R. Lahmann ${ }^{11}$, M.P.J. Landon ${ }^{20}$, W. Lange ${ }^{35}$, U. Langenegger ${ }^{36}$, A. Lebedev ${ }^{25}$, F. Lehner ${ }^{11}$, V. Lemaitre ${ }^{11}$, V. Lendermann ${ }^{8}$, S. Levonian ${ }^{11}$, M. Lindstroem ${ }^{21}$, B. List ${ }^{11}$, G. Lobo ${ }^{27}$, E. Lobodzinska ${ }^{6,41}$, V. Lubimov ${ }^{24}$, D. Lüke ${ }^{8,11}$, L. Lytkin ${ }^{13}$, N. Magnussen ${ }^{34}$, H. Mahlke-Krüger ${ }^{11}$, E. Malinovski ${ }^{25}$, R. Maraček ${ }^{17}$, P. Marage ${ }^{4}$, J. Marks ${ }^{14}$, R. Marshall ${ }^{22}$, G. Martin ${ }^{12}$, H.-U. Martyn ${ }^{1}$, J. Martyniak ${ }^{6}$, S.J. Maxfield ${ }^{19}$, S.J. McMahon ${ }^{19}$, T.R. McMahon ${ }^{19}$, A. Mehta ${ }^{5}$, K. Meier ${ }^{15}$, P. Merkel ${ }^{11}$, F. Metlica ${ }^{13}$, A. Meyer ${ }^{11}$, A. Meyer ${ }^{12}$, H. Meyer ${ }^{34}$, J. Meyer $^{11}$, P.-O. Meyer ${ }^{2}$, S. Mikocki ${ }^{6}$, D. Milstead ${ }^{11}$, J. Moeck ${ }^{26}$, R. $\mathrm{Mohr}^{26}$, S. Mohrdieck ${ }^{12}$, F. Moreau ${ }^{28}$, J.V. Morris ${ }^{5}$, D. Müller ${ }^{37}$, K. Müller ${ }^{11}$, P. Murín ${ }^{17}$, V. Nagovizin ${ }^{24}$, B. Naroska ${ }^{12}$, Th. Naumann ${ }^{35}$, I. Négrii ${ }^{23}$, P.R. Newman ${ }^{3}$, H.K. Nguyen ${ }^{29}$, T.C. Nicholls ${ }^{11}$, F. Niebergall ${ }^{12}$, C. Niebuhr ${ }^{11}$, Ch. Niedzballa ${ }^{1}$, H. Niggli ${ }^{36}$, D. Nikitin ${ }^{9}$, O. $\mathrm{Nix}^{15}$, G. Nowak 6 , T. Nunnemann ${ }^{13}$, H. Oberlack ${ }^{26}$, J.E. Olsson ${ }^{11}$, D. Ozerov ${ }^{24}$, P. Palmen ${ }^{2}$, V. Panassik ${ }^{9}$, C. Pascaud ${ }^{27}$, S. Passaggio ${ }^{36}$, G.D. Patel ${ }^{19}$, H. Pawletta ${ }^{2}$, E. Perez ${ }^{10}$, J.P. Phillips ${ }^{19}$, A. Pieuchot ${ }^{11}$, D. Pitzl ${ }^{36}$, R. Pöschl ${ }^{8}$, G. Pope ${ }^{7}$, B. Povh ${ }^{13}$, K. Rabbertz ${ }^{1}$, J. Rauschenberger ${ }^{12}$, P. Reimer ${ }^{30}$, B. Reisert ${ }^{26}$, H. Rick ${ }^{11}$, S. Riess ${ }^{12}$, E. Rizvi ${ }^{11}$, P. Robmann ${ }^{37}$, R. Roosen ${ }^{4}$, K. Rosenbauer ${ }^{1}$, A. Rostovtsev ${ }^{24,12}$, F. Rouse ${ }^{7}$, C. Royon ${ }^{10}$, S. Rusakov ${ }^{25}$, K. Rybicki ${ }^{6}$, D.P.C. Sankey ${ }^{5}$, P. Schacht ${ }^{26}$, J. Scheins ${ }^{1}$, 
S. Schleif ${ }^{15}$, P. Schleper ${ }^{14}$, D. Schmidt ${ }^{34}$, D. Schmidt ${ }^{11}$, L. Schoeffel ${ }^{10}$, V. Schröder ${ }^{11}$, H.-C. Schultz-Coulon ${ }^{11}$, B. Schwab ${ }^{14}$, F. Sefkow ${ }^{37}$, A. Semenov ${ }^{24}$, V. Shekelyan ${ }^{26}$,

I. Sheviakov ${ }^{25}$, L.N. Shtarkov ${ }^{25}$, G. Siegmon ${ }^{16}$, Y. Sirois ${ }^{28}$, T. Sloan ${ }^{18}$, P. Smirnov ${ }^{25}$, M. Smith ${ }^{19}$, V. Solochenko ${ }^{24}$, Y. Soloviev ${ }^{25}$, V .Spaskov ${ }^{9}$, A. Specka ${ }^{28}$, J. Spiekermann ${ }^{8}$, H. Spitzer ${ }^{12}$, F. Squinabol ${ }^{27}$, P. Steffen ${ }^{11}$, R. Steinberg ${ }^{2}$, J. Steinhart ${ }^{12}$, B. Stella ${ }^{32}$, A. Stellberger ${ }^{15}$, J. Stiewe ${ }^{15}$, U. Straumann ${ }^{14}$, W. Struczinski ${ }^{2}$, J.P. Sutton ${ }^{3}$, M. Swart ${ }^{15}$, S. Tapprogge ${ }^{15}$, M. Taševský ${ }^{30}$, V. Tchernyshov ${ }^{24}$, S. Tchetchelnitski ${ }^{24}$, J. Theissen ${ }^{2}$, G. Thompson ${ }^{20}$, P.D. Thompson ${ }^{3}$, N. Tobien ${ }^{11}$, R. Todenhagen ${ }^{13}$, P. Truöl ${ }^{37}$, G. Tsipolitis ${ }^{36}$, J. Turnau ${ }^{6}$, E. Tzamariudaki ${ }^{11}$, S. Udluft ${ }^{26}$, A. Usik ${ }^{25}$, S. Valkár ${ }^{31}$, A. Valkárováa ${ }^{31}$, C. Vallée ${ }^{23}$, P. Van Esch ${ }^{4}$, A. Van Haecke ${ }^{10}$, P. Van Mechelen ${ }^{4}$, Y. Vazdik ${ }^{25}$, G. Villet ${ }^{10}$, K. Wacker ${ }^{8}$, R. Wallny ${ }^{14}$, T. Walter ${ }^{37}$, B. Waugh ${ }^{22}$, G. Weber ${ }^{12}$, M. Weber ${ }^{15}$, D. Wegener ${ }^{8}$, A. Wegner ${ }^{26}$, T. Wengler ${ }^{14}$, M. Werner ${ }^{14}$, L.R. West ${ }^{3}$, S. Wiesand ${ }^{34}$, T. Wilksen ${ }^{11}$, S. Willard ${ }^{7}$, M. Winde ${ }^{35}$, G.-G. Winter ${ }^{11}$, C. Wittek ${ }^{12}$, E. Wittmann ${ }^{13}$, M. Wobisch ${ }^{2}$, H. Wollatz ${ }^{11}$, E. Wünsch ${ }^{11}$, J. Žáček ${ }^{31}$, J. Zálešák ${ }^{31}$, Z. Zhang ${ }^{27}$, A. Zhokin ${ }^{24}$, P. Zini' ${ }^{29}$, F. Zomer ${ }^{27}$, J. Zsembery ${ }^{10}$ and M. zurNedden ${ }^{37}$

\section{${ }^{1}$ I. Physikalisches Institut der RWTH, Aachen, Germany ${ }^{a}$}

${ }^{2}$ III. Physikalisches Institut der RWTH, Aachen, Germany ${ }^{a}$

3 School of Physics and Space Research, University of Birmingham, Birmingham, UK ${ }^{b}$

${ }^{4}$ Inter-University Institute for High Energies ULB-VUB, Brussels; Universitaire

Instelling Antwerpen, Wilrijk; Belgium ${ }^{c}$

${ }^{5}$ Rutherford Appleton Laboratory, Chilton, Didcot, $U K^{b}$

6 Institute for Nuclear Physics, Cracow, Poland ${ }^{d}$

7 Physics Department and IIRPA, University of California, Davis, California, USA ${ }^{e}$

8 Institut für Physik, Universität Dortmund, Dortmund, Germany ${ }^{a}$

9 Joint Institute for Nuclear Research, Dubna, Russia

${ }^{10}$ DSM/DAPNIA, CEA/Saclay, Gif-sur-Yvette, France

11 DESY, Hamburg, Germany ${ }^{a}$

12 II. Institut für Experimentalphysik, Universität Hamburg, Hamburg, Germany ${ }^{a}$

13 Max-Planck-Institut für Kernphysik, Heidelberg, Germany ${ }^{a}$

14 Physikalisches Institut, Universität Heidelberg, Heidelberg, Germany ${ }^{a}$

15 Institut für Hochenergiephysik, Universität Heidelberg, Heidelberg, Germany ${ }^{a}$

${ }^{16}$ Institut für experimentelle und angewandte Physik, Universität Kiel, Kiel, Germany ${ }^{a}$

17 Institute of Experimental Physics, Slovak Academy of Sciences, Košice, Slovak Republic $^{f, j}$

18 School of Physics and Chemistry, University of Lancaster, Lancaster, $U K^{b}$

19 Department of Physics, University of Liverpool, Liverpool, UK ${ }^{b}$

20 Queen Mary and Westfield College, London, UK ${ }^{b}$

21 Physics Department, University of Lund, Lund, Sweden ${ }^{g}$

${ }^{22}$ Department of Physics and Astronomy, University of Manchester, Manchester, UK ${ }^{b}$

${ }^{23}$ CPPM, Université d'Aix-Marseille II, IN2P3-CNRS, Marseille, France

${ }^{24}$ Institute for Theoretical and Experimental Physics, Moscow, Russia

${ }^{25}$ Lebedev Physical Institute, Moscow, Russia ${ }^{f, k}$

26 Max-Planck-Institut für Physik, München, Germany ${ }^{a}$

27 LAL, Université de Paris-Sud, IN2P3-CNRS, Orsay, France

28 LPNHE, École Polytechnique, IN2P3-CNRS, Palaiseau, France

${ }^{29}$ LPNHE, Universités Paris VI and VII, IN2P3-CNRS, Paris, France

${ }^{30}$ Institute of Physics, Academy of Sciences of the Czech Republic, Praha, Czech Republic $^{f, h}$ 
31 Nuclear Center, Charles University, Praha, Czech Republic ${ }^{f, h}$

32 INFN Roma 1 and Dipartimento di Fisica, Università Roma 3, Roma, Italy

33 Paul Scherrer Institut, Villigen, Switzerland

${ }^{34}$ Fachbereich Physik, Bergische Universität Gesamthochschule Wuppertal, Wuppertal, Germany ${ }^{a}$

${ }^{35}$ DESY, Institut für Hochenergiephysik, Zeuthen, Germany ${ }^{a}$

36 Institut für Teilchenphysik, ETH, Zürich, Switzerland ${ }^{i}$

37 Physik-Institut der Universität Zürich, Zürich, Switzerland ${ }^{i}$

38 Institut für Physik, Humboldt-Universität, Berlin, Germany ${ }^{a}$

39 Rechenzentrum, Bergische Universität Gesamthochschule Wuppertal, Wuppertal, Germany ${ }^{a}$

40 Vistor from Yerevan Physics Institute, Armenia

41 Foundation for Polish Science fellow

42 Dept. Fís. Ap. CINVESTAV, Mérida, Yucatán, México

a Supported by the Bundesministerium für Bildung, Wissenschaft, Forschung und Technologie, FRG, under contract numbers 7AC17P, 7AC47P, 7DO55P, 7HH17I, 7HH27P, 7HD17P, 7HD27P, 7KI17I, 6MP17I and $7 W T 87 P$

${ }^{b}$ Supported by the UK Particle Physics and Astronomy Research Council, and formerly by the UK Science and Engineering Research Council

${ }^{c}$ Supported by FNRS-FWO, IISN-IIKW

$d$ Partially supported by the Polish State Committee for Scientific Research, grant no. 115/E-343/SPUB/P03/002/97 and grant no. 2P03B 05513

e Supported in part by US DOE grant DE F603 91 ER40674

$f$ Supported by the Deutsche Forschungsgemeinschaft

$g$ Supported by the Swedish Natural Science Research Council

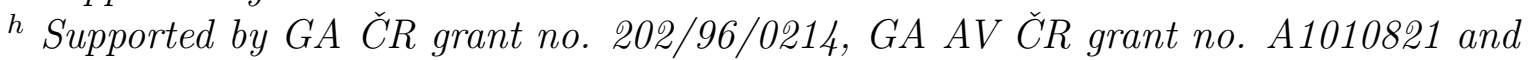
GA UK grant no. 17 ry

i Supported by the Swiss National Science Foundation

j Supported by VEGA SR grant no. 2/5167/98

${ }^{k}$ Supported by Russian Foundation for Basic Research grant no. 96-02-00019 


\section{Introduction}

The electron-proton collider HERA has made possible measurements of deeply inelastic scattering (DIS) in new kinematic regions: the regions of large four-momentum transfer $Q^{2}$ (up to $\left.Q^{2} \approx 10^{4} \mathrm{GeV}^{2}\right)$ and small Bjorken- $x\left(x \approx 10^{-4}\right)$. A diagram illustrating the QCD evolution of a low $x$ DIS event in the infinite momentum frame is shown in Fig. 1; a parton in the proton is able to undergo a QCD cascade resulting in several parton emissions before the final parton interacts with the virtual photon. These partons convert into hadrons which can then be detected and measured. The measurement of the hadronic final state between the proton remnant and the struck quark has been advocated over the past years as a technique for studying the dynamics of the QCD cascade in small $x$ DIS [1, 2, 3].

In this analysis data from the $\mathrm{H} 1$ experiment are used to study single particles and jets in the forward region, defined as polar angle, $\theta$, less than $25^{\circ}$ in the laboratory reference frame measured with respect to the proton beam direction. A complication in using the hadronic final state to investigate QCD evolution arises due to the soft hadronisation processes. It has been suggested that this can be reduced by studying forward jet production [1, 2, 3]. Such an analysis, based on a data sample containing approximately one tenth of the statistics used in this study, was reported in [4]. A similar analysis has been reported recently in [5]. Single high- $p_{T}$ particle production as a test of the underlying QCD dynamics has also been proposed [6]. A first analysis of transverse momentum $\left(p_{T}\right)$ and pseudorapidity $(\eta)$ spectra was made using charged particles in the pseudorapidity region $0.5<\eta<2.5$ in the $\gamma^{*} p$ centre of mass frame [7], accessing particles with angles down to $8^{\circ}$ in the laboratory frame. The advantages of studying single particles, rather than jets lie in their independence of any choice of jet finding algorithm and also the potential to reach smaller angles than would be possible with jets with broad spatial extent. For single particles however, fragmentation effects are expected to be more significant. Thus both measurements are complementary.

Several prescriptions for the QCD dynamics in the region of phase space towards the proton remnant have been proposed and are compared to the H1 data in this study. These include QCD parton evolution schemes such as the classical DGLAP (Dokshitzer-GribovLipatov-Altarelli-Parisi) [8 evolution, the low $x$ specific BFKL (Balitsky-Fadin-KuraevLipatov) [9] evolution equation and the CCFM (Ciafaloni-Catani-Fiorani-Marchesini) 10] evolution equation, which forms a bridge between the BFKL and DGLAP approaches. When the squared transverse momentum of a parton emitted in the ladder (Fig.1) is larger than $Q^{2}$ the resolved photon picture becomes applicable, in which case the photon can be considered to interact via its own resolved partonic component.

\section{QCD calculations and phenomenology}

DGLAP evolution, in which the relevant evolution parameter is $\ln Q^{2}$, has been successfully tested in large $Q^{2}$ processes and provides an excellent description of the scale dependence of the structure function of the proton. The BFKL evolution equation, for which the relevant evolution parameter is $\ln 1 / x$, describes scattering processes in QCD in the limit of large energies and fixed, but sufficiently large, momentum transfers. At lowest 


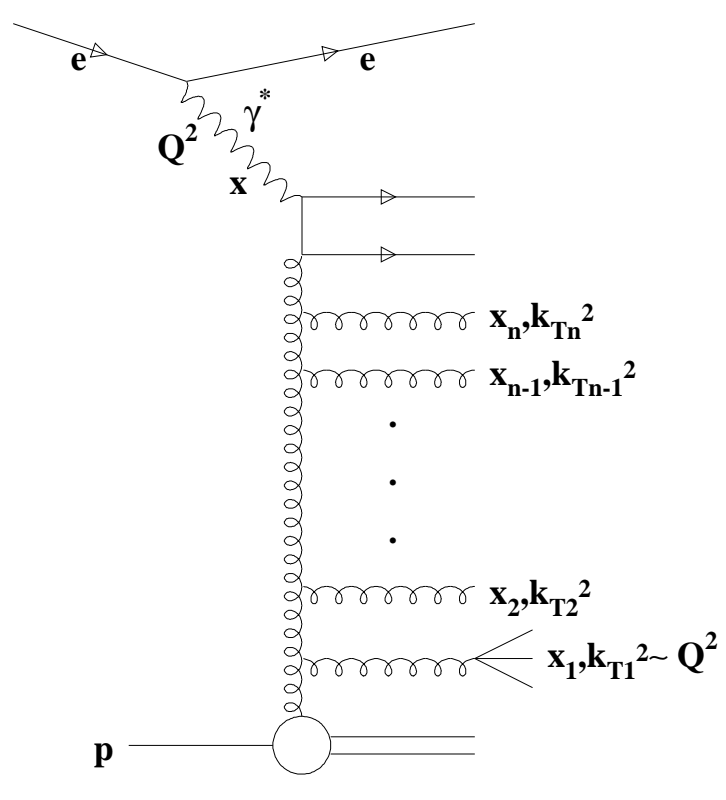

Figure 1: Parton evolution in the ladder approximation. The kinematics of forward jets in DIS events are indicated.

order the BFKL and DGLAP evolution equations effectively resum the leading logarithmic contributions $\alpha_{s} \ln 1 / x$ and $\alpha_{s} \ln Q^{2}$ respectively which, in an axial gauge, amounts to a resummation of ladder diagrams of the type shown in Fig. 1. Since it is expected that at small enough $x$ the $\ln 1 / x$ terms dominate the evolution, the BFKL equation should be applicable in this region. In the leading log DGLAP scheme the parton cascade follows a strong ordering in transverse momenta $k_{T n}^{2} \gg k_{T n-1}^{2} \gg \ldots \gg k_{T 1}^{2}$, while there is only a soft (kinematic) ordering for the fractional momenta $x_{n}<x_{n-1}<\ldots<x_{1}$. In the BFKL scheme, the cascade follows a strong ordering in fractional momentum $x_{n} \ll x_{n-1} \ll \ldots \ll x_{1}$, while there is no ordering in transverse momentum [11]. The transverse momenta perform a kind of random walk in $k_{T}$ space ; $k_{T i}$ is close to $k_{T i-1}$, but it can be both larger or smaller [12]. For the CCFM evolution equation the relevant quantity is the angular ordering in the cascade.

In a model that includes the resolved photon component in DIS the photon can be considered to interact via its own resolved partonic component. This internal photon structure leads to parton-parton scattering that can give rise to high $E_{T}$ jets, and emulate a random walk behaviour in $k_{T}$ [13. This is usually theoretically treated by ascribing a structure function to the photon and allowing DGLAP evolution to take place from partons in both the photon and the proton. The ladder shown in Fig.1 will then consist of two smaller ladders, one between the hard scattering and the photon, and one between the hard scattering and the proton. Consequently there is no longer a strict ordering in $k_{T}$ from the photon to the proton vertex, as obtained using the DGLAP picture without allowing virtual photon structure.

Predictions for final state observables are available from Monte Carlo models, based 
upon QCD phenomenology. LEPTO 6.5 [14] incorporates first order QCD matrix elements with matched leading-log DGLAP parton showers to simulate higher-order radiation (MEPS). For this model, the factorisation and renormalisation scales are set to $Q^{2}$. The MEPS scheme fails to account for the di-jet rates when $E_{\text {Tjet }}^{2} \geq Q^{2}$ [15, 16] in the kinematic range studied in this paper, although this is not unexpected since for $E_{\text {Tjet }}^{2} \gtrsim Q^{2}$ the parton from the proton is able to probe the structure of the photon. Using the concept of a resolved photon, as suggested above, the measured di-jet rates can be described [13, 16]. The Monte Carlo RAPGAP 2.06 [17] can include such a resolved photon contribution in addition to the standard "direct" process. For the study presented here the SAS-2D parametrisation [18] of the virtual photon parton densities was used, and $Q^{2}+p_{T}^{2}$, with $p_{T}$ the transverse momentum of the partons in the hard scattering, was used for the factorisation and renormalisation scales.

ARIADNE 4.08 [19] provides an implementation of the Colour Dipole Model (CDM) of a chain of independently radiating dipoles formed by emitted gluons [20]. Unlike that in LEPTO, the parton cascade generated in the CDM is not $k_{T}$ ordered [21]. Since all radiation is assumed to come from the dipole formed by the struck quark and the remnant, photon-gluon fusion events have to be added and are taken from the QCD matrix elements. The linked dipole chain (LDC) model [22] is a reformulation of the CCFM equation and redefines the separation of initial and final state QCD emissions using the CDM. This overcomes technical problems in calculating non-Sudakov form factors for parton splitting functions. Calculations of the hadronic final state based on this approach are available within the LDCMC 1.0 23] Monte Carlo which matches exact first order matrix elements with the LDC-prescribed initial and final state parton emissions. Both the LDCMC and ARIADNE use $p_{T}^{2}$ as the factorisation and renormalisation scales.

For the hadronisation of the partonic system, all of the above models use the Lund string model as implemented in JETSET [24]. All of these models are used with the GRV-HO 25 proton parton densities. QED corrections are determined with the Monte Carlo program DJANGO [26], using the CDM for the final state.

Alternatively, analytical BFKL calculations [27, 28] for forward jet production are available at the parton level. These calculations are based on asymptotic expressions derived from the BFKL equation at leading order (LO). In order to compare to single particle spectra a different approach has been adopted. The normalization of the calculation was fixed by comparing the prediction for the forward jet cross sections with the data, as reported in [28]. Once this normalization was fixed, predictions for particle spectra were made for the kinematic range given below, using fragmentation functions.

Also available are exact calculations of jet cross-sections at the parton level, at fixed order $\alpha_{s}^{2}$ in the strong coupling constant, for direct or pointlike interactions of the photon with the proton, as implemented in the DISENT [29] package. A comparison with DISENT for di-jet cross-sections has been reported in [16].

Background from photoproduction processes, those events for which $Q^{2} \sim 0$ in which the electron remains undetected in the beampipe and a fake electron is detected in the hadronic final state, has been studied using the PHOJET [30] Monte Carlo program. This generator contains LO QCD matrix elements for hard subprocesses, a parton showers model and a phenomenological description of soft processes. 


\section{The $\mathrm{H1}$ detector}

A detailed description of the $\mathrm{H} 1$ apparatus can be found elsewhere [31]. The following section briefly describes the components of the detector relevant to this analysis.

The hadronic energy flow and the scattered electron are measured with a liquid argon (LAr) calorimeter and a backward electromagnetic lead-scintillator calorimeter (BEMC) respectively. The LAr calorimeter [32 extends over the polar angle range $4^{\circ}<\theta<154^{\circ}$ with full azimuthal coverage. It consists of an electromagnetic section with lead absorbers and a hadronic section with steel absorbers. Both sections are highly segmented in the transverse and the longitudinal direction, in particular in the forward region of the detector, having about 45000 channels in total. The total depth of both calorimeters varies between 4.5 and 8 interaction lengths in the region $4^{\circ}<\theta<128^{\circ}$. Test beam measurements of the LAr calorimeter modules show an energy resolution of $\sigma_{E} / E \approx 0.50 / \sqrt{E[\mathrm{GeV}]} \oplus 0.02$ for charged pions [33]. The hadronic energy measurement is performed by applying a weighting technique in order to account for the noncompensating nature of the calorimeter. The absolute scale of the hadronic energy is presently known to $4 \%$, as determined from studies of the transverse momentum $\left(p_{T}\right)$ balance in DIS events with forward jets [34].

The BEMC (depth 22 radiation lengths or 1 interaction length) covers the backward region of the detector, $151^{\circ}<\theta<176^{\circ}$. The primary task of the BEMC is to trigger on DIS processes with $Q^{2}$ values ranging from 5 to $100 \mathrm{GeV}^{2}$, and to measure the scattered electron in DIS events. For scattered electrons with an energy larger than $11 \mathrm{GeV}$, as used in this analysis, the trigger efficiency is better than $99 \%$. The BEMC energy scale for electrons is known to an accuracy of $1 \%$ [35]. Its resolution is given by $\sigma_{E} / E=$ $0.10 / \sqrt{E[\mathrm{GeV}]} \oplus 0.39 / E[\mathrm{GeV}] \oplus 0.017[36]$.

The calorimeters are surrounded by a superconducting solenoid providing a uniform magnetic field of $1.15 \mathrm{~T}$ parallel to the beam axis in the tracking region. Charged particle tracks are measured in the central tracker (CT) covering the polar angular range $20^{\circ}<$ $\theta<160^{\circ}$ and the forward tracking (FT) system, covering the polar angular range $5^{\circ}<$ $\theta<25^{\circ}$. The CT consists of inner and outer cylindrical jet chambers, $z$-drift chambers and proportional chambers. The jet chambers, mounted concentrically around the beam line, cover a range of polar angle of $20^{\circ}<\theta<165^{\circ}$ and maximally provide 65 space points in the radial plane for tracks with sufficiently large transverse momentum. The achieved resolutions are $\sigma_{p_{T}} / p_{T} \approx 0.009 \cdot p_{T}[\mathrm{GeV}] \oplus 0.015$ and $\sigma_{\theta}=20 \mathrm{mrad}$ [31]. The FT provides measurements of tracks in the range $5^{\circ}<\theta<25^{\circ}$. This device has three identical sections, each containing a series of proportional, and radial and planar drift chambers, specifically arranged to facilitate triggering and track reconstruction at low polar angle. For tracks fitted to the interaction vertex the resolution in $p_{T}$ has been demonstrated to be $\sigma_{p_{T}} / p_{T} \approx 0.02 \cdot p_{T}[\mathrm{GeV}] \oplus 0.1$ and the angular resolution, $\sigma_{\theta}$, to be better than 1 $\operatorname{mrad}[37$.

A backward proportional chamber (BPC), in front of the BEMC with an angular acceptance of $155.5^{\circ}<\theta<174.5^{\circ}$ serves to identify electron candidates and to precisely measure their direction. Using information from the $\mathrm{BPC}$, the $\mathrm{BEMC}$ and the reconstructed event vertex the polar angle of the scattered electron is known to about 1 mrad.

The luminosity is measured using the reaction $e p \rightarrow e p \gamma$ with two $\mathrm{TlCl} / \mathrm{TlBr}$ crystal calorimeters, installed in the HERA tunnel. The electron tagger is located at $z=-33 \mathrm{~m}$ 
and the photon tagger at $z=-103 \mathrm{~m}$ from the interaction point in the direction of the outgoing electron beam.

\section{Data selection and corrections}

Experimental data for this analysis were collected by H1 during the 1994 running period, in which HERA collided $27.5 \mathrm{GeV}$ electrons with $820 \mathrm{GeV}$ protons. Integrated luminosities of the data samples used for the jets, $\pi^{0}$ and charged particle measurements are 2.8, 2.0 and $1.2 \mathrm{pb}^{-1}$ respectively. These differences arise due to the importance of selecting running periods with optimal experimental conditions for each of the measurements presented here.

DIS events are selected [35 via the scattered electron which is experimentally defined as a high energy cluster, i.e. a localised energy deposit in the BEMC, with a cluster radius less than $5 \mathrm{~cm}$ and with an associated hit in the $\mathrm{BPC}$. The scattered electron is required to satisfy $E_{e}^{\prime}>12 \mathrm{GeV}$ and $\theta_{\min }=156^{\circ}$ (for the particle analysis), or $E_{e}^{\prime}>11$ $\mathrm{GeV}$ and $\theta_{\min }=160^{\circ}$ (for the jet analysis), $\theta_{\min }<\theta_{e}<173^{\circ}$ where $E_{e}^{\prime}$ and $\theta_{e}$ are the energy and angle of the scattered electron. All results quoted here are corrected to their respective range. The kinematic variables are determined using information from the scattered electron: $Q^{2}=4 E_{e} E_{e}^{\prime} \cos ^{2}\left(\theta_{e} / 2\right)$ and $y=1-\left(E_{e} / E_{e}^{\prime}\right) \sin ^{2}\left(\theta_{e} / 2\right)$ where $E_{e}$ is the incident beam energy. The scaling variable Bjorken- $x$ is related to these quantities via the square of the centre of mass energy $s: x=Q^{2} /(y s)$. Further reduction of photoproduction background and the removal of events in which a high energy photon is radiated off the incoming electron are achieved by requiring $\sum_{j}\left(E_{j}-p_{z, j}\right)>35 \mathrm{GeV}$ [35], with the sum extending over all detected particles $j$ in the event, and by the requirement $y>0.1$, respectively. The events are required to have an event vertex which is within $30 \mathrm{~cm}$ of the nominal vertex position.

All distributions of data shown in this paper have been corrected bin-by-bin for detector effects, including geometrical acceptance, for QED radiative effects, and for the detection efficiencies of $\pi^{0}$ mesons, charged tracks and jets [38] respectively. The corrections were determined using events generated with the ARIADNE Monte Carlo program and a full simulation of the $\mathrm{H} 1$ detector response.

\subsection{Forward $\pi^{0}$ selection}

The $\pi^{0}$-mesons are measured using the dominant decay channel $\pi^{0} \rightarrow 2 \gamma$. The $\pi^{0}$ candidates are selected in the region $5^{\circ}<\theta_{\pi}<25^{\circ}$, where $\theta_{\pi}$ is the polar angle of the produced $\pi^{0}$. Candidates are required to have an energy of $x_{\pi}=E_{\pi} / E_{p}>0.01$, with $E_{p}$ the proton beam energy, and a transverse momentum with respect to the beam axis, $p_{T_{\pi}}$, greater than $1 \mathrm{GeV}$. Here, $p_{T_{\pi}}$ is taken to be equal to the transverse energy $E_{T, \pi}=E_{\pi} \sin \theta_{\pi}$. At the high $\pi^{0}$ energies considered here, the two photons from the decay cannot be separated, but appear as one object (cluster) in the calorimetric response. Photon induced showers are selected by measuring the shower shapes of candidate clusters. The selection criteria are based on the compact nature of electromagnetic showers as opposed to those of hadronic

\footnotetext{
${ }^{1}$ In 1994 the incident lepton at HERA was a positron although we use the generic name 'electron' for the incident and scattered lepton throughout this paper.
} 
origin. The very fine segmentation of the LAr calorimeter in the forward region (with a cell size of $3.5 \times 3.5 \mathrm{~cm}^{2}$ and four-fold longitudinal segmentation for the electromagnetic calorimeter) makes possible a detailed study of the transverse and longitudinal energy spread and the energy of each cluster. The high particle density in the forward direction leads to overlapping showers of electromagnetic and hadronic origin. Candidates of this type are largely rejected by the following requirements.

A $\pi^{0}$ candidate is required to have more than $90 \%$ of its energy deposited in the electromagnetic part of the LAr calorimeter. A "hot" core consisting of the most energetic group of continuous electromagnetic calorimeter cells of a cluster, which must include the hottest cell, is defined for each candidate [39]. More than 50\% of the cluster energy is required to be deposited in this core. The lateral spread of the shower is quantified in terms of lateral shower moments calculated relative to the shower's principal axis [39] and required to be less than $4 \mathrm{~cm}$ 40. The longitudinal shower shape is used as a selection criterion via the fraction of the shower's energy deposited in each layer of cells in the electromagnetic part of the calorimeter. The precise specifications of these layers can be found in [39]. The value obtained by subtracting the fraction of energy deposited in the fourth layer of cells from that in the second layer as seen from the interaction point is required to be larger than 0.4 , thereby selecting showers which start to develop close to the calorimeter surface and are well contained in the electromagnetic part of the calorimeter, as expected for showers of electromagnetic origin. With this selection $1673 \pi^{0}$ candidates are found in the kinematic range $5^{\circ}<\theta_{\pi}<25^{\circ}, x_{\pi}>0.01$ and $p_{T_{\pi}}>1 \mathrm{GeV}$ with a detection efficiency above $39 \%$.

Monte Carlo studies using a detailed simulation of the H1 detector predict that less than $20 \%$ of the $\pi^{0}$ candidates are due to other misidentified hadrons. These calculations also show that less than $10 \%$ of the candidates originate from secondary scattering of charged hadrons with passive material in the forward region, where the amount of material between the interaction point and the calorimeter surface is largest. This contamination is subtracted bin-by-bin. Background from photoproduction has been studied with the PHOJET Monte Carlo model, following the procedure used in the measurement of $F_{2}$ [35, and is found to make no significant contribution to the distributions shown.

\subsection{Charged particle selection}

Charged particles are selected using the forward (FT) and central (CT) tracking chambers, in the region $5^{\circ}<\theta_{\mathrm{cp}}<25^{\circ}$, where $\theta_{\mathrm{cp}}$ is the polar angle of the charged particle produced. Candidates are required to have an energy of $x_{\mathrm{cp}}=E_{\mathrm{cp}} / E_{p}>0.01$ and a transverse momentum with respect to the beam axis, $p_{T_{\mathrm{cp}}}$, greater than $1 \mathrm{GeV}$. Basic quality criteria are used to select well measured tracks originating from the primary interaction point. Tracks measured in the CT are required to have at least 10 associated hits and a radial track length of over $10 \mathrm{~cm}$. Selected tracks in the FT must have at least one planar segment confirmed by another segment reconstructed in either the planar or radial chambers. Furthermore, the impact parameter in the plane transverse to the beamline of the track from the primary vertex is required to be less than $5 \mathrm{~cm}$ when measured at the vertex position in $z$. Where a track has been found in both the FT and CT, a combined momentum measurement is used. For this measurement, as for the $\pi^{0}$-mesons, the high particle multiplicity in the forward direction constitutes the main challenge. It gives rise to a large flux of soft particles produced in secondary interactions 
in passive material both in the FT and preceding it, leading to a degradation of detection efficiency and resolution.

After the application of these cuts 2251 charged particles were selected for this study. The efficiency of reconstructing a central track and associating it to the primary event vertex is over 95\% 41]. The equivalent forward track efficiency is over 40\% [37 for tracks well contained within the FT. For tracks produced at low values of $\theta\left(\theta<10^{\circ}\right)$, an efficiency of $25 \%$ is obtained.

The proportion of selected charged particles that are produced in secondary interactions in the passive material in and around the $\mathrm{H} 1$ detector is negligible for central tracks. For the forward tracks it is estimated by a detailed simulation of the H1 detector to be maximally $10 \%$. This contribution is subtracted bin-by-bin. The assumed material distributions within the detector in the forward region have been verified by measuring the distribution of the rate of $\gamma \rightarrow e^{+} e^{-}$conversion pairs throughout the forward region of H1.

\subsection{Forward jet selection}

The region in which the forward jet measurements in this paper are made is chosen such that the phase space for jet production arising via DGLAP evolution is suppressed compared to that available in the case of BFKL evolution. This is achieved by requiring $k_{T \text { jet }}^{2} \approx Q^{2}$, where $k_{T \text { jet }}^{2}$ is the transverse momentum squared of the forward partonic jet. Due to the strong ordering in $k_{T}^{2}$, this leaves little room for DGLAP evolution. In addition, the momentum fraction of the jet, $x_{\text {jet }}=E_{\text {jet }} / E_{p}$, should be as large as possible, whereas the momentum fraction $x$ of the quark struck by the virtual photon should be small in order to maximize the phase space for jet production from BFKL evolution which is governed by the ratio $x_{\text {jet }} / x$. Here $E_{\text {jet }}$ and $E_{p}$ are the energies of the jet and the incoming proton respectively. The rate of events at small $x$ with a jet at high $x_{\text {jet }}$ should be much higher for the BFKL than for the DGLAP scenario, as was shown in [27].

A simple cone algorithm [38] is used to find jets in the H1 LAr calorimeter, requiring an $E_{T}$ larger than $3.5 \mathrm{GeV}$ in a cone of radius $R=\sqrt{\Delta \eta^{2}+\Delta \phi^{2}}=1.0$ in the space of pseudorapidity $\eta$ and azimuthal angle $\phi$ in the HERA frame of reference. This jet algorithm was found to give the best correspondence between jets at the detector and hadron level for this type of analysis 42. We have measured the cross-section for events which have a "forward" jet defined by $x_{\text {jet }}=E_{\text {jet }} / E_{p}>0.035,0.5<p_{T \text { jet }}^{2} / Q^{2}<2,7^{\circ}<\theta_{\text {jet }}<20^{\circ}$ and $p_{T \text { jet }}>3.5$ and $5 \mathrm{GeV}$, where $p_{T \text { jet }}$ is the transverse momentum of the jet, taken to be equal to $E_{T}$. A total of 1945 events contained at least one jet satisfying these criteria.

The transverse energy flow around the forward jet axis, averaged over all selected events, is shown versus $\Delta \eta$ and $\Delta \phi$ with respect to the reconstructed jet axis in Fig. 2, for various $p_{T \text { jet }}$ ranges. Distinct jet profiles are observed which are reasonably described by the DJANGO model with full detector simulation.

Backgrounds from photoproduction and radiative events (for which the current jet is boosted into the forward region) have been studied with Monte Carlo and with real data. The proportion of radiative events was reduced by requiring a cluster with energy larger than $0.5 \mathrm{GeV}$ and pseudorapidity between -1.3 and 1.5 units of rapidity away from the forward jet axis, to tag the hadronic activity of the current quark. A fraction of the 

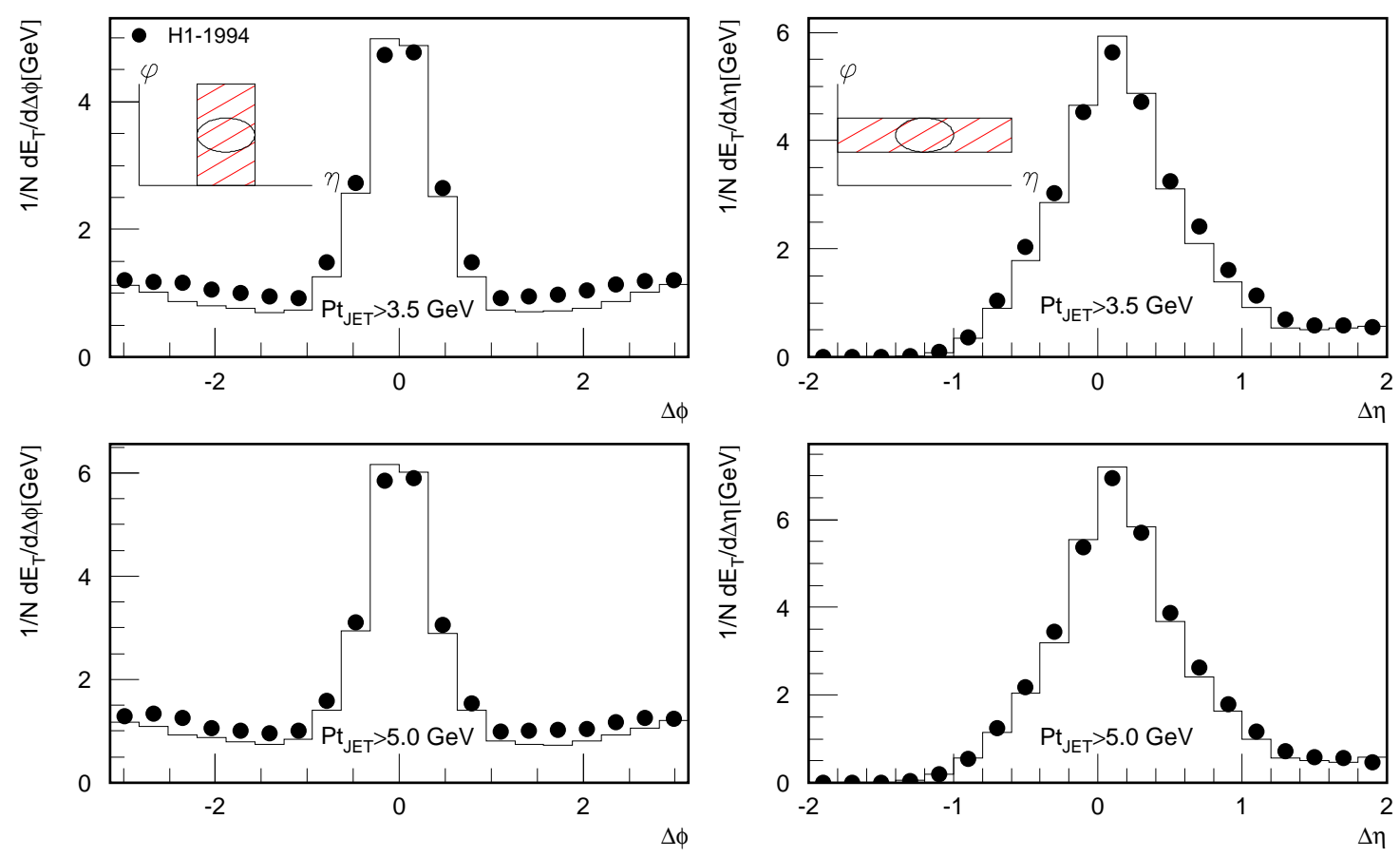

Figure 2: The average transverse energy flow around the forward jet axis over all events is shown for two different minimum $p_{T \text { jet }}$ values as a function of $\Delta \phi$ (left ), integrated over $|\Delta \eta|<1$ and as a function of $\Delta \eta$, (right), integrated over $|\Delta \phi|<1 \mathrm{rad}$, as shown by the insets in the top figures. Here $\Delta \eta$ and $\Delta \phi$ are measured with respect to the reconstructed jet axis. Also shown is the DJANGO expectation with full H1 detector simulation.

background events can be experimentally measured (tagged) using the detectors of the luminosity system: for radiative events with a photon emitted collinearly with the incident electron, the photon can be detected with the photon tagger; for photoproduction events the electron which is scattered through a small angle can be detected in the electron tagger. Using data samples with tagged events it is possible to check the Monte Carlo based estimates of the photoproduction background. This is found to be less than $3 \%$ and subtracted from the data presented below.

The forward jet analysis has also been repeated using an unfolding procedure 34 based on the Bayes unfolding method [43]. The results agree very well with the bin-to-bin corrected data.

\section{Results}

In Fig. 3, single particle spectra are presented as a function of Bjorken- $x$ for $\pi^{0}$-mesons and charged particles. The $\pi^{0}$-mesons are shown for $5^{\circ}<\theta_{\pi}<25^{\circ}$ in two bins of $x_{\pi}\left(0.01<x_{\pi}\right.$ $<0.015$ and $\left.0.015<x_{\pi}<0.15\right)$ for $p_{T_{\pi}}>1 \mathrm{GeV}$ and for the higher threshold of $p_{T_{\pi}}>2$ $\mathrm{GeV}$ in the $x_{\pi}$ range $0.01<x_{\pi}<0.15$. All distributions are normalized to the number of DIS events, $N$, in the kinematic region specified in section 1 and with the additional requirement that Bjorken- $x$ is in the range $0.0002<x<0.00235$. The full errors are the quadratic sum of the statistical (inner error bars) and systematic uncertainties. For the $\pi^{0}$ measurements the systematic errors contain the model dependence of the correction, 

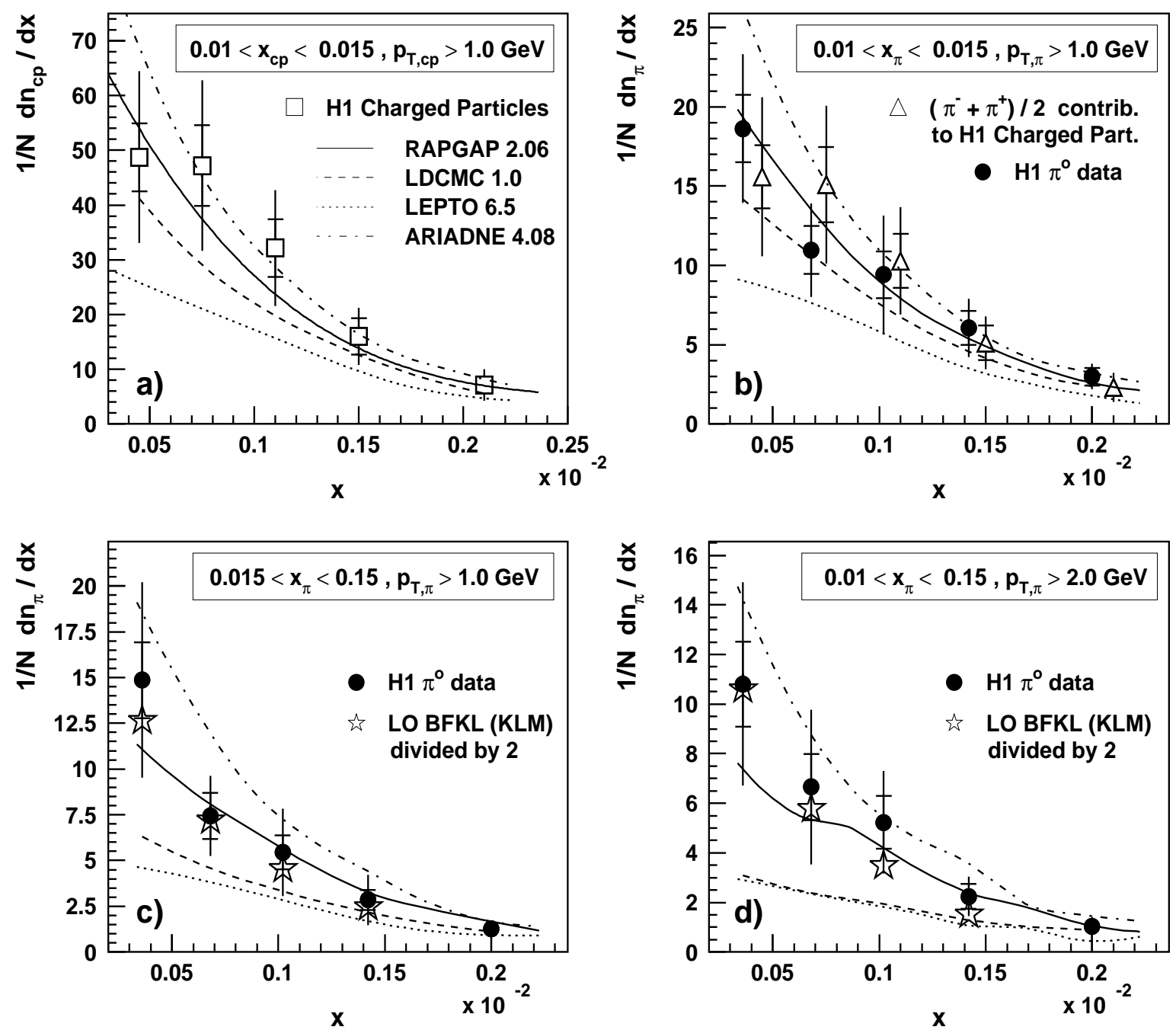

Figure 3: The single particle spectra in Bjorken- $x$ are shown for charged particles and $\pi^{0}$-mesons produced in the polar angle range $5^{\circ}<\theta<25^{\circ}$. In the upper right plot ARIADNE with JETSET was used to calculate the contribution of charged pions to the charged particle measurement which makes possible, when divided by two, a direct consistency check of the two measurements. For comparison, four different Monte Carlo models are overlaid, as well as an analytical calculation labelled BFKL(KLM) based on [28. $n_{\pi}$ is the number of $\pi^{0}$-mesons and $N$ is the number of DIS events that fall into the specified kinematic range. The full errors are the quadratic sum of the statistical (inner error bars) and systematic uncertainties.

estimated from the difference in correction factors obtained using LEPTO and ARIADNE, which leads to errors of between 20-30\%; a $4 \%$ variation of the electromagnetic energy scale in the forward region gives a $5 \%$ uncertainty; the variation of $\pi^{0}$ selection cuts contributes $5-10 \%$ systematic error; and the variation of $\pi^{0}$ acceptance also produces a $5-10 \%$ uncertainty.

The dominant source of systematic uncertainty for the charged particle measurements stems from the dependence of the FT efficiency on the forward particle multiplicity, which leads to a large systematic error due to the model dependence of the correction procedure. 
This gives typical point-to-point errors of between $10 \%$ and $25 \%$ in the analysis presented here. These numbers were evaluated using simulated LEPTO and ARIADNE events. Discrepancies between the simulation and the FT detector response give rise to further point-to-point systematic errors of about 15\%. Other sources of systematic effects, such as the BEMC energy scale uncertainty and photoproduction background are included in the errors but are small compared to those quoted above.

The data in Fig. 3 clearly show that the production of $\pi^{0}$-mesons with high $p_{T}$ increases towards small Bjorken- $x$. The binning was chosen to match the $x$ resolution. Only for the lower bin in $x_{\pi}$ and $p_{T}$ is a measurement of charged particles available. To check the consistency of both measurements the charged particles results have been converted to charged pions spectra using the charged pion fraction predicted by ARIADNE with JETSET, which describes the charged particle data well. From isospin symmetry the neutral pion spectrum is expected to be half of the total charged pion spectrum. The resulting charged pion spectrum, divided by a factor two, is overlaid on Fig. 33b, and is found to be in good agreement with the $\pi^{0}$ measurement. The model predictions shown will be discussed below.

The forward jet data for $p_{T \text { jet }}>3.5 \mathrm{GeV}$ and $p_{T \text { jet }}>5 \mathrm{GeV}$ are shown as a function of Bjorken- $x$ in the range $0.0001<x<0.004$ in Fig. $\$$. In the case that there are two jets in the event which satisfy the jet selection criteria (approximately 1\% of the forward jet events, see below), the jet with the largest $p_{T \text { jet }}$ was taken. It was shown that with the HERA kinematics and detector constraints, which determine the smallest reachable angle for $\theta_{\text {jet }}$, the largest differences between BFKL and fixed order calculations are expected for low values of $p_{T \text { jet}}$, namely around $3.5 \mathrm{GeV}$ 44. Reducing this value further leads to excessively large hadronisation corrections as predicted by ARIADNE and dubious jet selections. However, to study the sensitivity of this study to increasing $p_{T \text { jet }}$, crosssections with $p_{T \text { jet }}>5 \mathrm{GeV}$ have also been measured. The data points in Fig. 囵 present the cross-section as measured in the given $x$ bin for the kinematic and jet cuts defined above. The bin purity, i.e. the fraction of events in a bin in $x$ with a jet at the detector level which also have a jet at the hadron level in that bin, is between $25 \%$ and $50 \%$ for the lower $p_{T \text { jet }}$ threshold value and somewhat larger for the higher $p_{T \text { jet }}$ threshold value.

The data shown in Fig. 1 clearly rise with decreasing $x$, except for the smallest $x$ bin, for which the rise is hidden due to the narrow kinematic region left with the present cuts. The total cross-section for forward jets with $p_{T \text { jet }}>3.5 \mathrm{GeV}$ in the defined kinematic range is $531 \pm 17(\text { stat. })_{-89}^{+82}($ syst. $)$ pb.

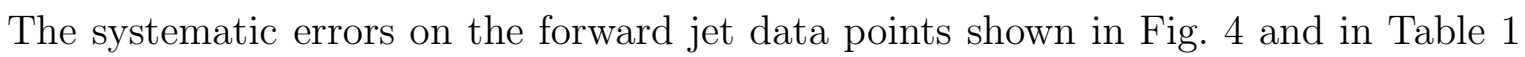
are dominated by the $4 \%$ uncertainty of the hadronic energy scale, leading to shifts in the jet rates of $12 \%$. Another important systematic contribution results from the dependence on the model used to correct the data and is up to $7 \%$ (the data shown are corrected with the ARIADNE model). The energy scale of the BEMC affects the cross-section by $3-4 \%$ in Figs. 1 and 5. Other systematic effects have been found to be small compared to the above.

The Monte Carlo models described above are compared to the data in Fig. 4. The data rise faster with decreasing $x$ than LDCMC and LEPTO, which are based on the CCFM equation and LO DGLAP dynamics respectively. The prediction of ARIADNE is slightly below the jet data and is slightly above the single particle data. ARIADNE does however reproduce the rise towards low $x$. Unlike LEPTO, this model does not include 

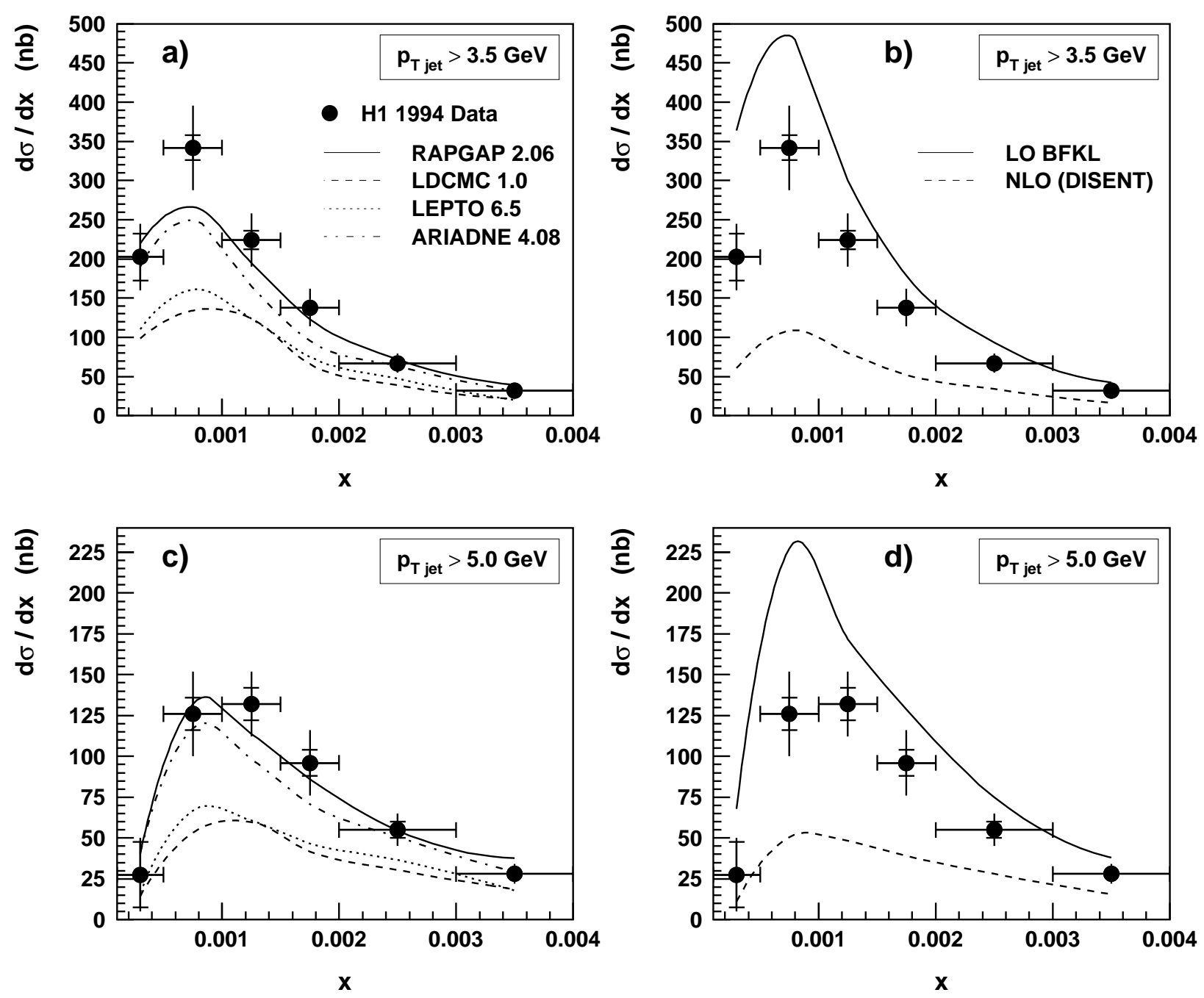

Figure 4: The forward jet cross-section as a function of Bjorken- $x$ for two $p_{\text {Tjet }}$ cuts: $3.5 \mathrm{GeV}$ and $5.0 \mathrm{GeV}$. The errors shown are the statistical and systematic uncertainties added in quadrature. In (a) and (c) the curves are model calculations (full line RAPGAP, dashed line LDCMC, dotted line LEPTO and the dashed dotted line ARIADNE). In (b) (d) the full lines are analytic LO BFKL calculations at the parton level (no jet algorithm was applied), the dashed lines $O\left(\alpha_{s}^{2}\right)$ calculations using DISENT with a cone algorithm applied.

a $k_{T}$ ordered cascade and it has been suggested that these calculations will be close to the BFKL prediction [21]. It has however also been hypothesised [45 that the sizeable QCD radiation predicted by this model in the forward region stems not from the lack of a $k_{T}$ ordered cascade but from an unconventional enhancement of QCD-Compton processes at low $x$ in which a hard gluon is radiated after the parton-photon interaction. The ARIADNE predictions also depend on the photon and target size extension parameters used in the calculations?.

The RAPGAP model, which is based on LO DGLAP dynamics but has in addition a

\footnotetext{
${ }^{2}$ The default values $\operatorname{PARA}(10)=1.0$ and $\operatorname{PARA}(14)=1.0$ have been used. Changing these parameters to 1.5 ( 0.5$)$ reduces (increases) the cross-sections by approximately $15-30 \%$
} 


\begin{tabular}{|c|c|}
\hline Event Sample & Forward jet cross-section [pb] \\
\hline H1 1994 Data & $531 \pm 17(\text { stat. })_{-89}^{+82}$ (syst. $)$ \\
\hline ARIADNE 4.08 & 425 \\
\hline RAPGAP 2.06 & 491 \\
\hline LEPTO 6.5 & 259 \\
\hline LDCMC 1.0 & 262 \\
\hline
\end{tabular}

Table 1: Cross-sections for $\mathrm{H} 1$ data and QCD calculations for inclusive forward jets satisfying $x_{\text {jet }}>0.035,0.5<p_{T \text { jet }}^{2} / Q^{2}<2,7^{\circ}<\theta_{\text {jet }}<20^{\circ}$ and $p_{T \text { jet }}>3.5 \mathrm{GeV}$ in the kinematic range $E_{e}^{\prime}>11 \mathrm{GeV}, 160^{\circ}<\theta_{e}<173^{\circ}$ and $y>0.1$.

contribution from resolved photons, gives a good description of both the single particle and jet data. The RAPGAP Monte Carlo allows the study of the scale dependence of the result. Sensible variations of the choice of scale used ${ }^{[}$in the leading order calculation were found to reduce the resolved photon cross-section calculations by up to $40 \%$. The direct cross-section is more stable with respect to the choice of scale and changes by less than $10 \%$. A comparison of the integrated forward jet cross-section for data and for these Monte Carlo models is given in Table 1 1 . The Monte Carlo cross-sections are based on event samples with numbers of events which are a factor 2 to 3 larger than those in the data samples.

The forward jet data, shown in Fig. 14 are also compared with recent analytical LO BFKL calculations [27] and a fixed order $O\left(\alpha_{s}^{2}\right)$ jet calculation at the parton level. No jet algorithm is used for the BFKL calculation while a cone algorithm 46] has been used for the fixed order calculation. The BFKL calculation is above the measurements for the data with both $p_{T \text { jet }}$ thresholds of 3.5 and $5 \mathrm{GeV}$. The fixed order prediction for direct interactions of the photon with the proton is well below the data and very close to the so called "Born" calculation in [27], for which no gluon emissions are allowed in the BFKL ladder. The latter corresponds to the quark box diagram seen by the photon, and one single gluon at the low end of the diagram as is explained in [27]. Variations of the fixed order cross section predictions of about $40 \%$ are found when the scale $\left(Q^{2}\right)$ is varied by a factor of four.

Hadronisation corrections and kinematic constraints (for the BFKL calculations) may change the predictions made at the parton level. The former are unfortunately strongly model dependent and at the lowest $x$ values LEPTO and ARIADNE predict a change of the jet cross-section due to hadronisation by factors (number of jets at parton level/ number of jets at hadron level) of 1.0 and 0.7 for $p_{T \text { jet }}>3.5 \mathrm{GeV}$, and 1.5 and 1.1 for $p_{T \text { jet }}>5$ $\mathrm{GeV}$ respectively. The kinematic constraints are expected to lower the predicted crosssections [47]. It should also be noted that the BFKL calculations contain a typical power growth of the cross-section with $x$ with a power of about 0.5 . Recently, next-to-leading order BFKL calculations for the inclusive cross-sections have become available [48] which demonstrate large corrections to this power, substantially reducing it. The question of how these large corrections will change the numerical BFKL predictions for the HERA forward jets cross-sections has not yet been addressed; in fact a recent paper 44] argues that the next-to-leading order reduction of the BFKL exponent might be somewhat

\footnotetext{
${ }^{3}$ The scale was changed from $p_{T}^{2}+Q^{2}$ to $4 p_{T}^{2}+Q^{2}$.
} 
smaller than naively expected. Furthermore, the next-to-next-to-leading order corrections may also be large. In view of these uncertainties, the interpretation of possible deviations of experimental data from fixed order matrix element calculations as being due to BFKL effects has become more difficult.

BFKL calculations folded with fragmentation functions are also available for the highest momentum $\pi^{0}$ bins. This prediction is a factor of two above the data although the shape is in well described. This is shown in Fig. 3 in which the prediction, divided by two [50], is compared to the data. Despite this normalisation discrepancy the calculations are seen to describe well the $x$ dependence of the $\pi^{0}$ measurements.
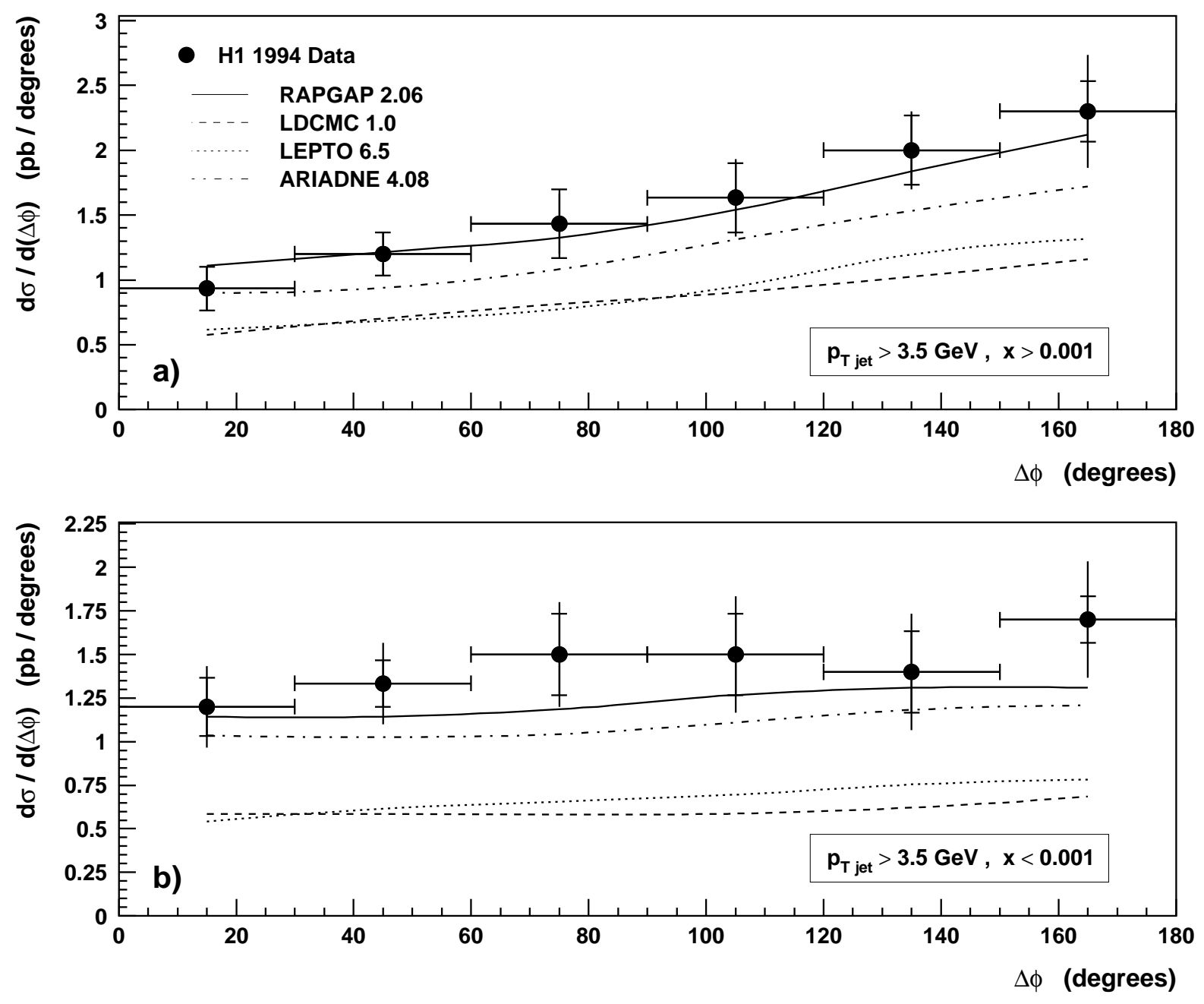

Figure 5: The azimuthal correlation $\Delta \phi$ (in degrees) between the jet and the scattered electron, for two different $x$ regions. The curves are model calculations (full line RAPGAP, dashed line LDCMC, dotted line LEPTO and the dashed dotted line ARIADNE).

It has been suggested that a study of the azimuthal correlation $\Delta \phi$ between the scattered electron and the forward jet [27] may be a sensitive discriminant between the BFKL and DGLAP evolution schemes. A stronger decorrelation at low $x$ is expected in the 


\begin{tabular}{|c|c|}
\hline Event Sample & Forward di-jet cross-section $(\mathrm{pb})$ \\
\hline H1 1994 Data & $6.0 \pm 0.8(\text { stat. })_{-3.1}^{+3.2}$ (syst.) \\
\hline ARIADNE 4.08 & 6.7 \\
\hline RAPGAP 2.06 & 6.0 \\
\hline LEPTO 6.5 & 1.8 \\
\hline LDCMC 1.0 & 2.1 \\
\hline
\end{tabular}

Table 2: Cross-sections for $\mathrm{H} 1$ data and different model calculations for forward di-jet events, each satisfying $x_{\text {jet }}>0.035,0.5<p_{T \text { jet }}^{2} / Q^{2}<2,7^{\circ}<\theta_{\text {jet }}<20^{\circ}$ and $p_{T \text { jet }}>3.5 \mathrm{GeV}$ in the kinematic range $E_{e}^{\prime}>11 \mathrm{GeV}, 160^{\circ}<\theta_{e}<173^{\circ}$ and $y>0.1$.

BFKL case than in that of DGLAP due to the dilution of the original correlation resulting from multi-gluon emission. The data are shown in Fig. 5 for the smaller and higher $x$ regions. The data exhibit the expected levelling of the distribution when $x$ decreases although all models follow this tendency, suggesting that this is not a very sensitive variable in this kinematic range.

Of the 1945 forward jet events selected, 52 have a second jet which fulfill the forward jet criteria. This corresponds to a total cross-section of $6.0 \pm 0.8$ (stat.) \pm 3.2 (syst.) pb, or roughly $1 \%$ of the forward jet cross-section. Recent LO BFKL numerical studies at the parton level [51] predict that $3 \%$ of events will have two or more forward jets. We have also checked that this discrepancy between the data and the BFKL calculation remains if the ratio of $Q^{2}$ to $p_{T \text { jet }}^{2}$ is relaxed to $0.5<p_{T \text { jet }}^{2} / Q^{2}<5$. However, the BFKL calculations, as before, include neither the effects of hadronisation nor those of jet algorithms. Table 2 compares the forward di-jet cross-section in data with the QCD models. ARIADNE and RAPGAP give di-jet rates in agreement with the data, while the other models predict lower values and, owing to the current precision of the measurement, cannot yet be excluded.

\section{Summary}

Measurements of the cross sections for the production of forward jets and high transverse momentum single particles in low $x$ DIS have been presented. Models whose dynamics are based on different schemes for QCD evolution have been compared with the results. The conclusions using the forward jet and the single particle measurements are in agreement.

Models implementing the traditional DGLAP evolution and including only direct photon interactions grossly underestimate the amount of perturbative radiation required. Also a $O\left(\alpha_{s}^{2}\right)$ jet calculation predicts less jet production. Models which include also the resolved photon component successfully describe all the data presented. BFKL calculations at leading order describe the strong rise of the forward single jet and particle cross-sections at low $x$. While BFKL effects are expected to be prominent in this low $x$ domain complete BFKL calculations have still to be performed at next-to-leading order before the data can be interpreted in this light. 
Predictions of a model based on an implementation of CCFM evolution, which should smoothly interpolate between the DGLAP and BFKL regimes, give a poor description of all measured distributions. Calculations implementing the Colour Dipole Model provide sufficient QCD radiation to match the data.

\section{Acknowledgements}

We are grateful to the HERA machine group whose outstanding efforts have made and continue to make this experiment possible. We thank the engineers and technicians for their work in constructing and now maintaining the $\mathrm{H} 1$ detector, our funding agencies for financial support, the DESY technical staff for continual assistance, and the DESY directorate for the hospitality which they extend to the non-DESY members of the collaboration. We would like to thank J. Bartels, J. Kwiecinski, S. Lang and W. J. Stirling for useful discussions.

\section{References}

[1] A. H. Mueller, Nucl. Phys. B (Proc. Suppl.) 18C (1990) 125; J. Phys. G17 (1991) 1443.

[2] J. Kwieciński, A.D. Martin, P.J. Sutton, Phys. Rev. D46 (1992) 921.

[3] J. Bartels, A. De Roeck, M. Loewe, Z. Phys. C54 (1992) 635;

W. K. Tang, Phys. Lett. B278 (1992) 363.

[4] H1 Collab., S. Aid et al. Phys. Lett. B356 (1995) 118.

[5] ZEUS Collab., J. Breitweg et al., DESY-98-050, hep-ex/9805016 (1998).

[6] M. Kuhlen, Phys. Lett. B382 (1996) 441.

[7] H1 Collab., C. Adloff et al., Nucl. Phys. B485 (1997) 3.

[8] Yu. L. Dokshitzer, Sov. Phys. JETP 46 (1977) 641;

V. N. Gribov and L. N. Lipatov, Sov. J. Nucl. Phys. 15 (1972) 438 and 675;

G. Altarelli and G. Parisi, Nucl. Phys. 126 (1977) 297.

[9] E. A. Kuraev, L. N. Lipatov and V. S. Fadin, Sov. Phys. JETP 45 (1972) 199;

Y. Y. Balitsky and L. N. Lipatov, Sov. J. Nucl. Phys. 28 (1978) 822.

[10] M. Ciafaloni, Nucl. Phys. B296 (1988) 49;

S. Catani, F. Fiorani and G. Marchesini, Phys. Let. 234B (1990) 339;

S. Catani, F. Fiorani and G. Marchesini, Nucl. Phys. B336 (1990) 18.

[11] A. H. Mueller, Columbia preprint CU-TP-658 (1994) and Cargese Summer Inst. (1994) 87.

[12] J. Bartels and H. Lotter, Phys. Lett. B309 (1993) 400.

[13] H. Jung, L. Jönsson, H. Küster, hep-ph/9805396 (1998), DESY-98-51. 
[14] G. Ingelman, Proc. of the HERA workshop, eds. W. Buchmüller and G. Ingelman, Hamburg (1992) vol. 3, p. 1366.

[15] H1 Collab., C. Adloff et al. Phys. Lett. B415 (1997) 418.

[16] H1 Collab., C. Adloff et al., DESY 98-076, submitted to Europ. Phys. J. C.

[17] H. Jung, Comp. Phys. Comm. 86 (1995) 147; (for update see http://wwwh1.desy.de/ jung/rapgap/rapgap.htm]).

[18] G. A. Schuler, T. Sjostrand, Phys. Lett. B376 (1996) 193.

[19] L. Lönnblad, Comp. Phys. Comm. 71 (1992) 15.

[20] G. Gustafson, Phys. Lett. B175 (1986) 453;

B. Andersson, G. Gustafson, L. Lönnblad and U. Pettersson, Z. Phys. C43 (1989) 625 ;

G. Gustafson, U. Pettersson, Nucl. Phys. B306 (1988) 746.

[21] L. Lönnblad, Z. Phys. C65 (1995) 285;

A. H. Mueller, Nucl. Phys. B415 (1994) 373;

L. Lönnblad, Z. Phys. C70 (1996) 107.

[22] B. Andersson, G. Gustafson, J. Samuelsson, Nucl. Phys. B467 (1996) 443.

B. Andersson, G. Gustafson, H. Kharraziha, J. Samuelsson, Z. Phys. C71 (1996) 613.

[23] H. Kharraziha, L. Lönnblad, hep-ph/9709424 (1997).

[24] T. Sjöstrand, Comp. Phys. Comm. 39 (1986) 347;

T. Sjöstrand and M. Bengtsson, Comp. Phys. Comm. 43 (1987) 367;

T. Sjöstrand, CERN-TH-6488-92 (1992).

[25] M. Glück, E. Reya and A. Vogt, Z. Phys C67 (1995) 433.

[26] G. A. Schuler and H. Spiesberger, Proc. of the HERA Workshop, Vol 3. , Eds. W. Buchmüller and G. Ingelman, DESY 1992, 1419.

[27] J. Bartels et al., Phys. Lett. B384 (1996) 300.

[28] J. Kwiecinski, S. C. Lang and A. D. Martin, hep-ph/9707240;

J. Kwiecinski, S. C. Lang and A. D. Martin, Phys. Rev. D55 (1997) 1273.

[29] S. Catani, M.H. Seymour, Nucl. Phys. B485 (1997) 291, Erratum-ibid. B510 (1997) 503.

[30] R. Engel, Proceedings of the XXIXth Rencontre de Moriond (1994) 321.

[31] H1 Collab., I. Abt et al., Nucl. Instr. and Methods A386 (1997) 310.

H1 Collab., I. Abt et al., Nucl. Instr. and Methods A386 (1997) 348.

[32] H1 Calorimeter Group, B. Andrieu et al., Nucl. Instr. and Meth. A336 (1993) 460.

[33] H1 Calorimeter Group, B. Andrieu et al., Nucl. Instr. and Meth. A336 (1993) 499.

[34] E. M. Lobodzinska, PhD. Thesis, Krakow University, 1997 (unpublished). 
[35] H1 Collab., S. Aid et al., Nucl. Phys. B470 (1996) 3.

[36] H1 BEMC group, J. Ban et al., Nucl. Inst. and Meth. A372 (1996) 399.

[37] S. Burke et al., Nucl. Instr. and Meth. A373 (1996) 227.

[38] J. G. Contreras Nuno, PhD. Thesis, Dortmund University 1997 (unpublished).

[39] H1 Calorimeter Group, B. Andrieu et al., Nucl. Instr. and Meth. A344 (1994) 492.

[40] T. Wengler, PhD. Thesis, Heidelberg University, to appear.

[41] H1 Collab., S. Aid et al., Z. Phys. C72 (1996) 573.

[42] J. Kurzhöfer, PhD. Thesis, Dortmund University 1995 (unpublished).

[43] G. D. Agostini, Nucl. Instr. Meth. A362 (1995) 487.

[44] J. Bartels, A. De Roeck and M. Wüsthoff, Proceedings of the workshop Future Physics at HERA, Eds. G. Ingelman, A. De Roeck, R. Klanner, p. 598 (1996).

[45] J. Rathsman, Phys. Lett. B393 (1997) 181.

[46] CDF Collab., F. Abe et al., Phys. Rev. D45 (1992) 1448.

[47] W. J. Stirling, talk at the workshop on Deep Inelastic Scattering, DIS98, Brussels, to appear in proceedings.

[48] V.S. Fadin and L. N. Lipatov, Phys. Lett. B429 (1998) 127.

[49] D. A. Ross, hep-ph/9804332 (1998), SHEP-98-06.

[50] A. Martin and S. Lang, private communication.

[51] J. Kwiecinski, C. A. M. Lewis and A. D. Martin, Phys. Rev. D57 (1998) 496. 